\author{
Military Technical College \\ Kobry El-Kobbah, \\ Cairo, Egypt.
}

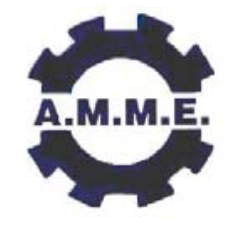

$13^{\text {th }}$ International Conference on Applied Mechanics and Mechanical Engineering.

\title{
EFFECTS OF A DIRECTED CO-FLOW ON A TURBULENT METHANE JET
}

\author{
IMINE* B., SENOUCI* M. and BOULENOUAR*N
}

\begin{abstract}
In the present study, the effects of a directed co-flow on the process of Methane jet have been investigated numerically. The directed co-flow preserves its axial symmetry at the inlet and its direction varies between $\alpha=+20^{\circ}$ and $\alpha=-20^{\circ}$. In addition, the $k-\varepsilon$ model is used to investigate effects of the variable density in axisymmetric turbulent jet. The results obtained indicate that the directed co-flow with positive angles enhances considerably the mixing.
\end{abstract}

\section{KEYS WORDS}

Jet, co-flow, variable density, turbulence and numerical simulation.

\section{NOMENCLATURE}

F Average value of the mixture fraction

$D_{j} \quad$ Jet diameter at the inlet

$\mathrm{T}$ Value of the temperature

$\mathrm{K}$ Kinetic Energy of the turbulence....

$\mathrm{Ku}$ Decay rate of the longitudinal velocity

$\cup \quad$ Mean axial velocity

$\mathrm{V}$ Mean radial velocity

$R \rho$ Density ratio $\left(\rho, / \rho_{a n b}\right)$

$\tilde{f}^{\prime \prime 2}$ Variance of mixture fraction

Faculté de Mécanique, Laboratoire de Mécanique Appliquée - U.S.T.O. B.P 1505 USTO Oran El Mnaouer - Algeria- Fax 0021341342199 - imine b@yahoo.fr. 


\author{
Greek symbols \\ $\varepsilon \quad$ Rate of dissipation of $\mathrm{K}$ \\ $\alpha$ Angle of co-flow deviation \\ $\varepsilon_{f}$ Scalar dissipation rate \\ Indices and exponents \\ a Co flow \\ c Centreline \\ j Jet

\section{Superscripts} \\ $\widetilde{\Phi} \quad$ Favre average of $\Phi$ \\ $\bar{\Phi}$ Conventional average of $\Phi$ \\ Favre fluctuation \\ Conventional fluctuation
}

\title{
INTRODUCTION
}

The variation of the density can be caused either by a mixing of several flows with different species or by a temperature gradient between an air jet and a surrounding atmosphere. Since the sixties, the influence of the co-flows surrounding the turbulent jets was the object of many studies. In order to reduce the turbulence intensities on the nozzle edges or to have good boundary conditions for modeling, a co-flow is often used. An analytical study on the jets with co-flow has been investigated by Abramovich [1]. He showed the presence of three essential areas: initial, principal and transition areas. When the flow is confined, the process of the co-flow entrained by the jet is modified. A considerable pressure gradient generates phenomena of recirculation. Curtet [2] was interested by this phenomena and proposed a parameter of similarity called parameter of Craya-Curtet, which is formulated in the literature of the variable density by Steward and Guruz [3]. He showed for a value of this number higher than 0.8 the phenomenon of recirculation is avoided, Djeridane [4], Ruffin [5], Raddaoui [6], Lucas [7], Page' [8] and Gazzah [9] were concentrated on the later study for choice of the co-flow velocity. In the experimental study of Djeridane [4], two configurations of coflow were adopted, one with circular section and the other with square section, it showed that the axial behavior of the longitudinal mean velocity and its fluctuation is independent of the confinement form.

In most cases, the designer wishes to use a new configuration that ensures rapid mixing of the jet with the co-flow. In the present study, the effects of a directed co-flow on the mixing process with variable density have been investigated numerically. The directed co-flow preserves its axial symmetry at the inlet and its direction varies between $\alpha=+20^{\circ}$ and $20^{\circ}$ and the negative angles are directed towards the axis of symmetry as shown in Fig. 1.

\section{CONSERVATION EQUATIONS AND TURBULENCE MODELS}

In the mathematical description of the conservation equations, all variables, except the pressure and the density which are always computed according to Reynolds average, are Favre average[10]. This quantity is defined as 


$$
\widetilde{\Phi}=\frac{\overline{\rho \Phi}}{\bar{\rho}}
$$

The turbulent jet with variable density is a monophasic flow of Newtonian fluid which can be regarded as a perfect gas. The general form of the transport equations for an axisymmetric flow is:

$$
\frac{\partial}{\partial x}(\rho U \phi)+\frac{1}{r} \frac{\partial}{\partial r}(r \rho V)=\frac{\partial}{\partial x}\left(\Gamma_{\phi} \frac{\partial \phi}{\partial x}\right)+\frac{1 \partial}{r \partial r}\left(r \Gamma_{\phi} \frac{\partial \phi}{\partial r}\right)+S_{\phi}
$$

where $\phi, \Gamma_{\varphi}$ and $S_{\phi}$ are respectively the transportable quantity, the diffusion term and the source term. The system of equations governing a confined jet is presented in Table 1.

The mean density can be obtained from the mean mixture fraction using the equation of state, Sanders et al. [26]. With constant pressure, this leads to:

$\frac{1}{\bar{\rho}}=\frac{F}{\rho_{j}}+\frac{1-F}{\rho_{a}}$.

\section{First order turbulence models}

These models are based on the hypothesis of Boussinesq, which it connect the Reynolds stress $\left(\overline{\rho u_{i}^{\prime \prime} u_{j}^{\prime \prime}}\right)$ to the mean velocity gradient and to turbulent viscosity. In the same way, the fluxes of the turbulent scalars $\overline{\rho u_{i}^{\prime \prime} f^{\prime \prime}}$ can be connected to the mean scalar gradients using the suitable diffusion coefficients. For the $k$ - $\varepsilon$ model the eddy viscosity is computed by $v_{t}=C_{\mu} k^{2} / \varepsilon$, where $C_{\mu}$ is a model constant.

The source term $S_{\Phi}$ are given in Table 2 and the turbulent diffusion coefficient is defined as $D_{\phi}=\frac{v_{t}}{\sigma_{\phi}}$. The turbulent energy production, the scalar fluctuation production and the buoyancy production are defined respectively as:

$$
\begin{aligned}
& P=-\bar{\rho} u^{\prime \prime} v^{\prime \prime} \frac{\partial U}{\partial r} \\
& P_{\widetilde{f}^{\prime \prime 2}}=2 \bar{\rho} \frac{\partial F}{\partial r} \frac{\partial F}{\partial r} \\
& G=-\bar{\rho} \beta_{e} \widetilde{f}^{\prime \prime 2} u^{\prime \prime} f^{\prime \prime}
\end{aligned}
$$

The constants used in this work are given in Table 3.

\section{NUMERICAL METHOD}

The equations describing a confined turbulent flow are of elliptic convection-diffusion. 
These equations are solved by a finite volume method as described by Patankar and Spalding [11]. It consists in the integration of these equations in a control volume. For the numerical solution of these equations a computer code was developed. The terms of the differential on the volume interfaces are obtained by a second order upwind scheme. The pressure velocity coupling is achieved by the SIMPLE algorithm of Patankar and Spalding [11]. The grid extends gradually in all directions in order to take into account of the jet development in the co-flowing. The mesh in the two directions become wider when one moves away from the emission section of the jet, but at the same time the gradients become less significant and the precision is thus not influenced.

\section{RESULTS}

Different deviations of co-flow around a $\mathrm{CH} 4$ jet are investigated. The experimental operating conditions of Pagé [8] are used for this calculation. In the figure 2, the inverse normalized centreline velocity ( $\mathrm{Uj}-\mathrm{Ua}$ ) / (Uc-Ua) with different values of $\alpha:\left(-20^{\circ} \leq \alpha \leq+20^{\circ}\right)$ is plotted. A similar effect is observed on the slowness of the centreline longitudinal velocity decay. This later reminds constant for all deviations along the potential core and start to increase at $\mathrm{X} / \mathrm{Dj}=6$ as a linear function. In figure 3 , for several deviations, the slope of the linear reciprocal velocity evolution $K_{u}$ is plotted. It is observed that this evolution is linear and can be correlated by the following relation:

$$
K_{u}=0.00141 \alpha+0.0749 .
$$

In the far field, it is noticed, that the centreline velocity (Uc-Ua)/(Uj-Ua), for $\alpha=+20^{\circ}$, decays about $29.7 \%$ faster than in the case of $\alpha=0^{\circ}$. In contrast, the centreline velocity in the case of $\alpha=-20^{\circ}$ decays about $20.3 \%$ more slowly than in the case $\alpha=0^{\circ}$. The axial mean mixture fraction presents the most important characteristic parameter of mixing jet. The figure 4 shows the influence of the directed air co-flow on the reciprocal axial mean mixture fraction of $\mathrm{CH} 4$ jet . After the end of the potential core, the inverse mean mixture fraction becomes a linear function of the distance from the exit jet and is defined by the following equation:

$\frac{1}{\mathrm{Fc}}=\frac{1}{\mathrm{~K}_{\mathrm{f}}} \frac{\mathrm{X}}{\mathrm{D}_{\mathrm{j}}}$

The same operating conditions of Pagé [8] and three deviations of air co-flow $\alpha=+20^{\circ}$, $0^{\circ}$ and $-20^{\circ}$ are used to investigate the evolution of the mean mixture fraction $\mathrm{CH} 4$ jet at different cross sections (figure 5). The cross sections at $X / D_{j}=3$ shows the initial development of a thin shear layer. It is observed that, at $x / D_{j} \geq 6$, the profiles take Gaussian forms which are flattened more and more with distance $\mathrm{X}$. The increase of $\alpha$ generates a greater jet radial expansion, accompanied by a diminution in the mixture fraction on the centreline jet. A co flow directed with positive angles enhances strongly the mixing.

In combustion applications, the stoi-chiometry and global equivalence ratio are used to define a mixing efficiency. However, in mixing jet flows, parameters such as the jet halfwidth or the scalar axial decay are used in the literature. These parameters only reflect 
the local behaviour of the mixing process. Sanders et al [12] have proposed a new definition of a mixing efficiency which is defined by the following expression:

$$
I / I_{0}=\left(1 / \rho_{j} D_{j}^{3}\right) \int_{0}^{\infty} \bar{\rho} \widetilde{\mathrm{F}} \mathrm{dA}
$$

with $d A=r d r$ where $r$ is the radial distance to the axis and $I_{0}$ represents the mass quantity at the emission section.

The mixing efficiency can be regarded as a measurement of the mass quantity from the jet. Figure 6 represents the axial evolution of this parameter for a mixture $\left(\mathrm{CH}_{4}\right.$-air $)$ and for different angles. The effect of the directed co-flow on the mixing efficiency is clearly observed. It is noted that for $0 \leq X / D_{j} \leq 45$, this parameter increases when the angle of deviation increases up to the value of $X / D_{j}=45$ where all the curves have the same mixing efficiency value.

In Fig. 7, the effect of the directed co flow on the centreline turbulent kinetic energy of $\mathrm{CH} 4$ jet with different values of $\alpha$ is shown. In the case of $\alpha=0^{\circ}$ the obtained result agree well with the experimental data of Pagé [8]. It was observed just downstream of the inlet jet as shown in figure 7 , a growth followed by a decrease of the turbulent kinetic energy. This growth is more significant when the angle of deviation $\alpha$ is large. Moreover, the maximum of the centreline turbulent kinetic energy is reached at the same axial position of the potential core for all cases tested and is large when the angle of co-flow deviation $\alpha$ augments.

\section{CONCLUSION}

In this present work, a numerical study of effects of the directed co-flow around a turbulent binary mixing jet with variable density has been investigated. A calculation of the centreline values of longitudinal velocity, mixture fraction, mixing efficiency and turbulent kinetic energy has been presented. The prediction of the present calculation agrees reasonably well with the very recent experimental study in the case of a co flow not directed. $\left(\alpha=0^{\circ}\right)$. The introduction of a directed co-flow with positive angles made it possible to obtain very good performance of mixing. According to results obtained, this configuration can be used as application in the combustion chamber or in particular in the burners to improve the mixing process more in order to ensure a good combustion.

\section{REFERENCES}

1. Abramovich G.N (, 1963)., The theory of turbulent jets, Cambridge MA, the MIT press.

2. Curtet $\mathbf{R}$ (1957) Contribution à l'étude théorique des jets de révolution. Extrait des comptes rendus de l'académie des sciences, Vol. 244, pp. 1450-1453.

3. Steward F.R., Guruz A.G. (1977) Aerodynamic of a confined jet with variable density.

Comb..Sci. Tech., Vol. 16, pp. $29-45$.

4. Djeridane $\mathbf{T}$ (1994) Contribution à l'étude expérimentale de jets turbulents axisymétriques à densité variable, Thèse de doctorat, université d'Aix-Marseille II. 
5. Ruffin E (1994) Etude de jets turbulents à densité variable à l'aide de modèles de transport au second ordre. Thèse de doctorat, Université d'Aix Marseille.

6. Raddaoui M (1997) Modélisation numérique et etude expérimentale du mélange d'echelles dans un jet turbulent confiné, Thèse de doctorat, Université d'Aix Marseille.

7. Lucas J. F (1998), Analyse du champ scalaire au sein d'un jet turbulent axisymétrique à densité variable, Thèse de doctorat, Université d'Aix Marseille.

8. Pagé J. (1998) Contribution à l'étude des jets turbulents axisymétrique à masse volumique variable. Thèse de doctorat, Université d'Orléans.

9. Gazzah M. H. (2002) Contribution à l'étude d'un jet turbulent à masse volumique variable, Thèse de doctorat, Ecole Nationale d'Ingénieurs de Monastir, Tunisie.

10. Favre A., Kovasnay L.S.G., Dumas R., Gaviglio J., Coantic M (1976) La turbulence en mécanique des fluides : bases théoriques et expérimentales, methods statistiques. Paris:Bordas, Gaulthier-Villars. 411 p. ISBN 2-04-003566-4.

11. Patankar S.V, Spalding D.B (1970) Heat and Mass Transfer in Boundary Layers. Intertext, London.

12 Sanders J. P, H., Sarh B, Gökalp I (1997), Variable density effects in axisymmetric isothermal turbulent jets : a comparison between a first-and a second-order turbulence model, Int. J. Heat Mass Transfer, Vol. 40 (4), pp. 823842.

Table 1: Conservation equations.

\begin{tabular}{|c|c|c|}
\hline$\phi$ & $\Gamma_{\phi}$ & $S_{\phi}$ \\
\hline 1 & 0 & 0 \\
\hline$U$ & $\mu_{e f f}$ & $-\frac{\partial p}{\partial x}+\frac{\partial}{\partial x}\left(\mu_{e f f} \frac{\partial U}{\partial x}\right)+\frac{1}{r \partial} \frac{\partial}{r}\left(r \mu_{e f f} \frac{\partial V}{\partial x}\right)$ \\
\hline$V$ & $\mu_{e f f}$ & $-\frac{\partial p}{\partial r}+\frac{\partial}{\partial x}\left(\mu_{e f f} \frac{\partial U}{\partial r}\right)+\frac{1}{r \partial} \frac{\partial}{r}\left(r \mu_{e f f} \frac{\partial V}{\partial r}\right)-2 \mu_{e f f} \frac{V}{r^{2}}$ \\
\hline $\mathrm{F}$ & $\mu_{e f f} / \sigma_{f}$ & 0 \\
\hline
\end{tabular}

Table 2: Source terms in the general equations of $k-\varepsilon$

\begin{tabular}{|c|c|}
\hline$\phi$ & $S_{\Phi}$ \\
\hline$k$ & $P+G-\bar{\rho} \varepsilon$ \\
\hline$\varepsilon$ & $\left.\frac{\varepsilon}{k}\left(C_{\varepsilon, 1} P+C_{\varepsilon, 3} G\right)-C_{\varepsilon, 2} \bar{\rho} \varepsilon\right)$ \\
\hline$\widetilde{f}^{\prime \prime 2}$ & $P_{\widetilde{f}^{\prime 2}}-\bar{\rho} \varepsilon_{f}$ \\
\hline
\end{tabular}

Table 3: Constants of the model $k-\varepsilon$ used in the present study

\begin{tabular}{|l|l|l|l|l|l|l|l|}
\hline$C \mu=0.09$ & $C_{\varepsilon, 1}=1.44$ & $C_{\varepsilon, 2}=1.92$ & $C_{\varepsilon, 3}=1.92$ & $\sigma_{k}=1.0$ & $\sigma_{\varepsilon}=1.3$ & $\sigma_{f}=0.7$ & $\sigma_{\widetilde{f}^{\prime 2}}=0.7$ \\
\hline
\end{tabular}




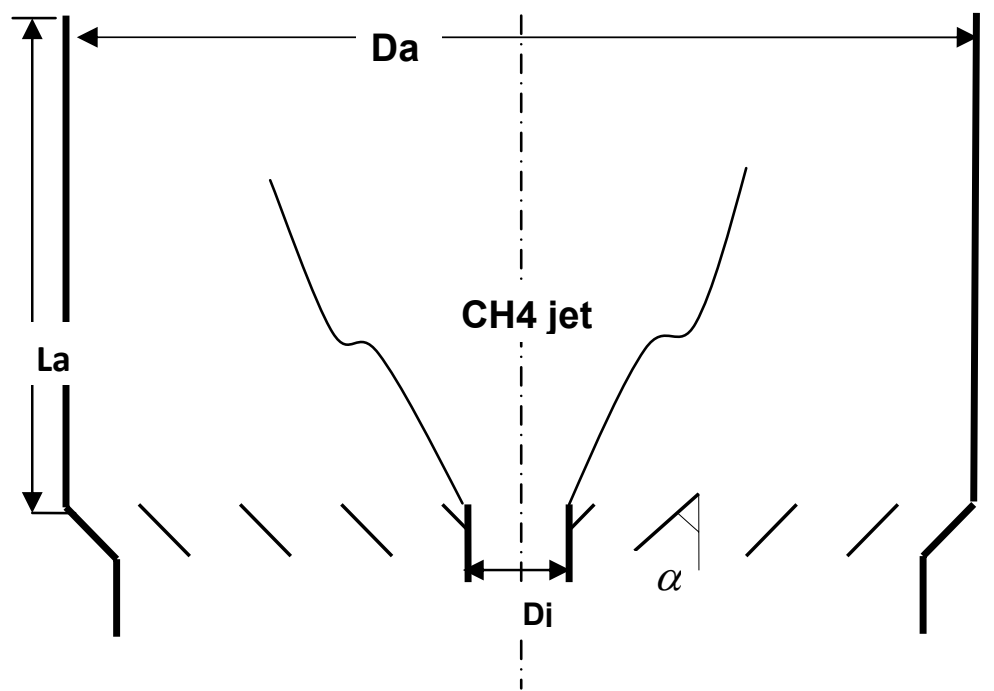

Fig. 1 Calculation domains in the case of positive angle.

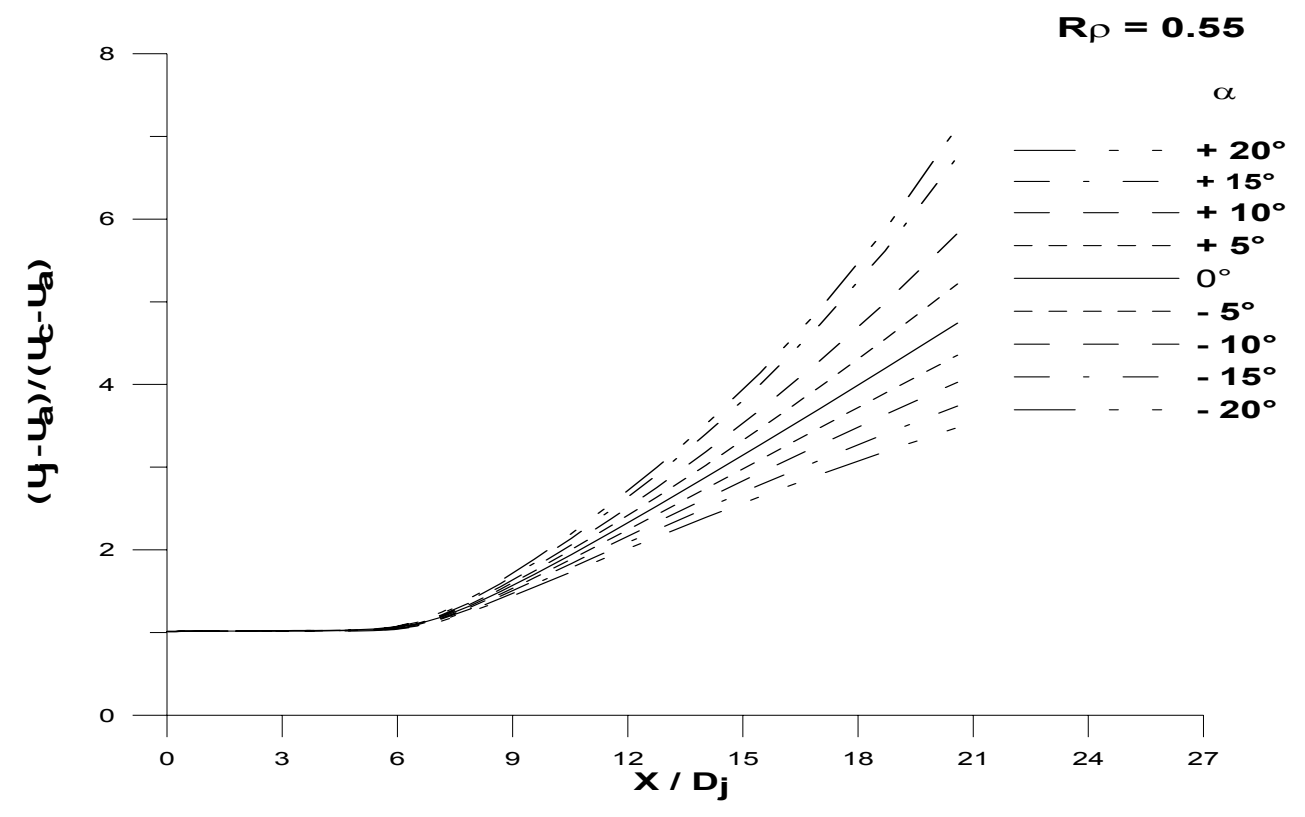

Fig. 2 Variation of axial velocity along centreline. 


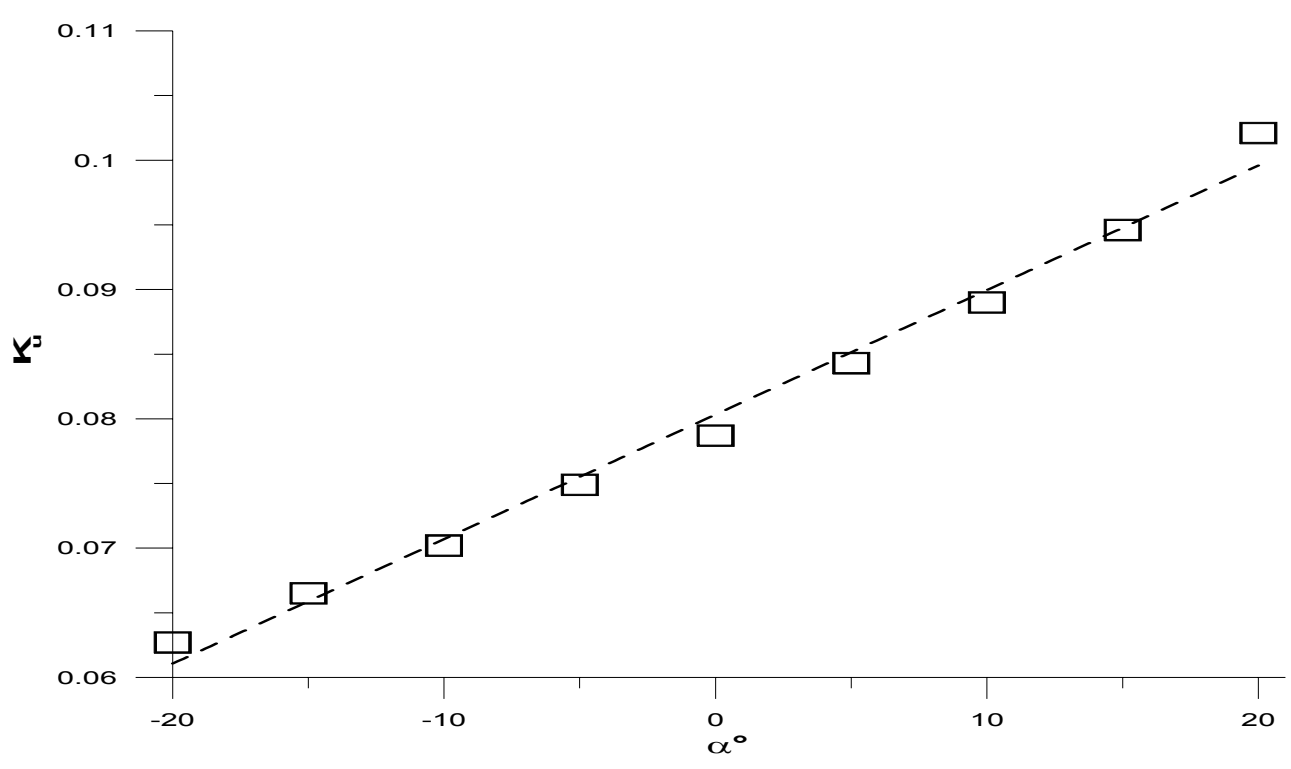

Fig. 3 Evolution of the decay rate of the inverse longitudinal velocity.

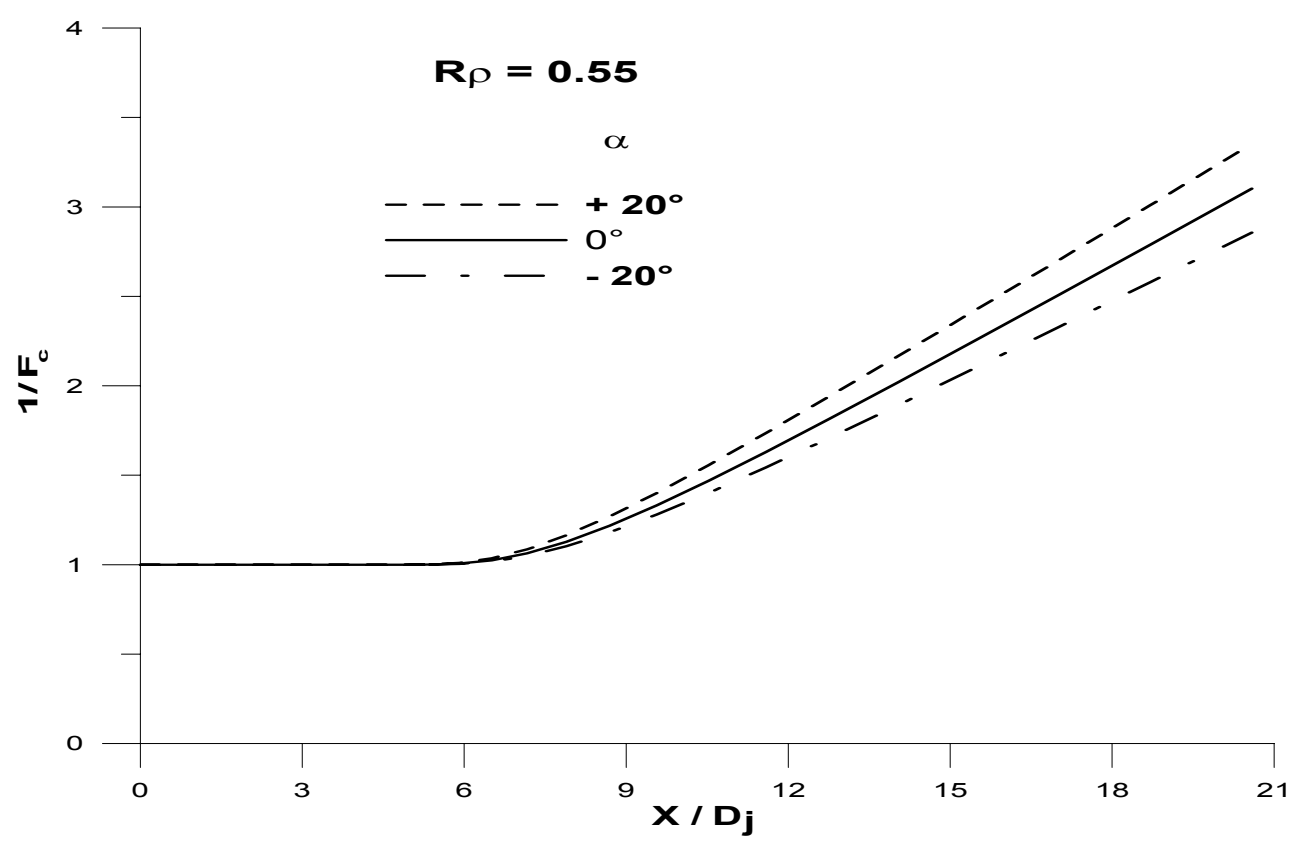

Fig. 4 Reciprocal mean mixture along centreline. 

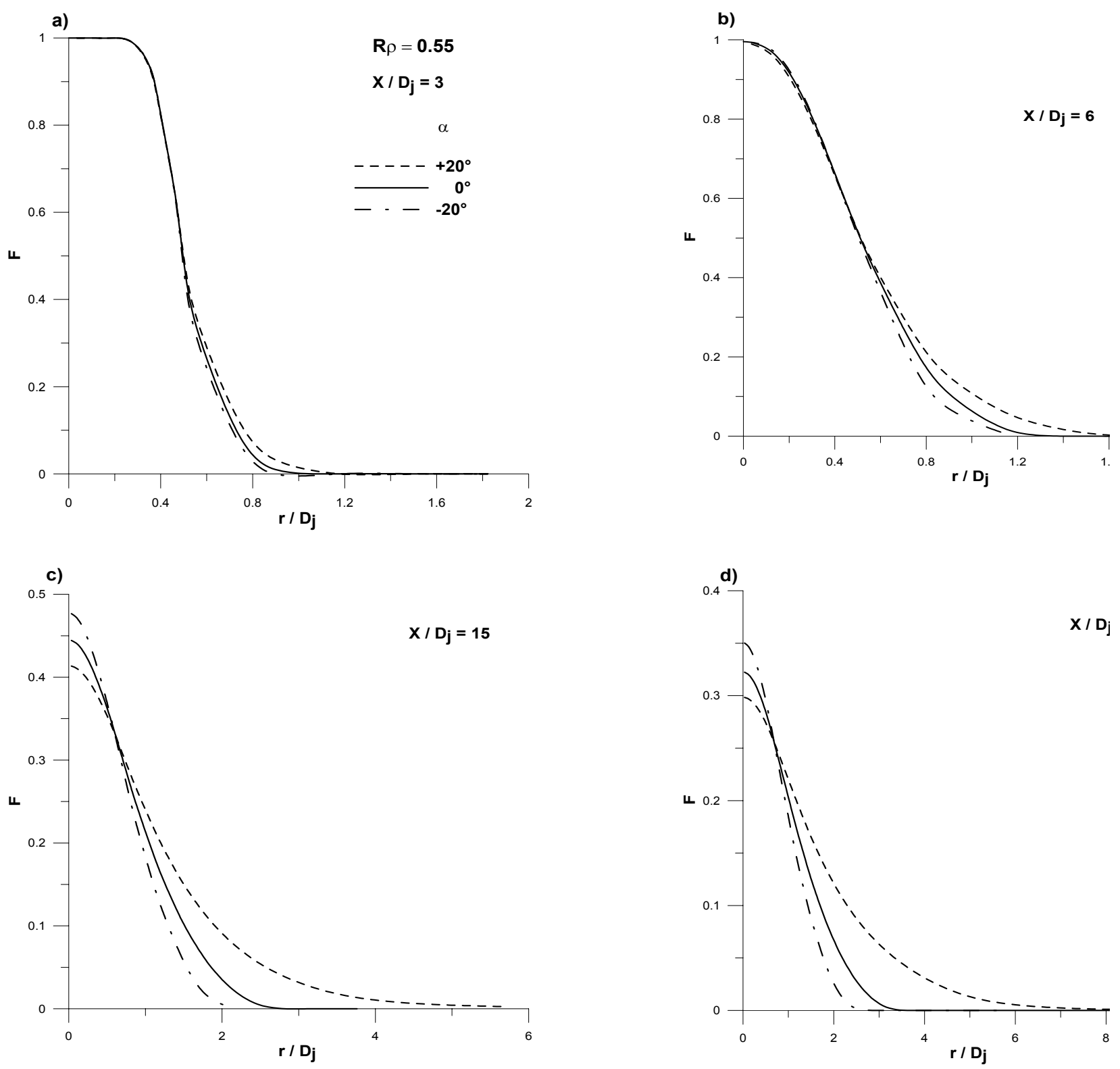

Fig. 5 Radial profiles of the mean mixture fraction. 


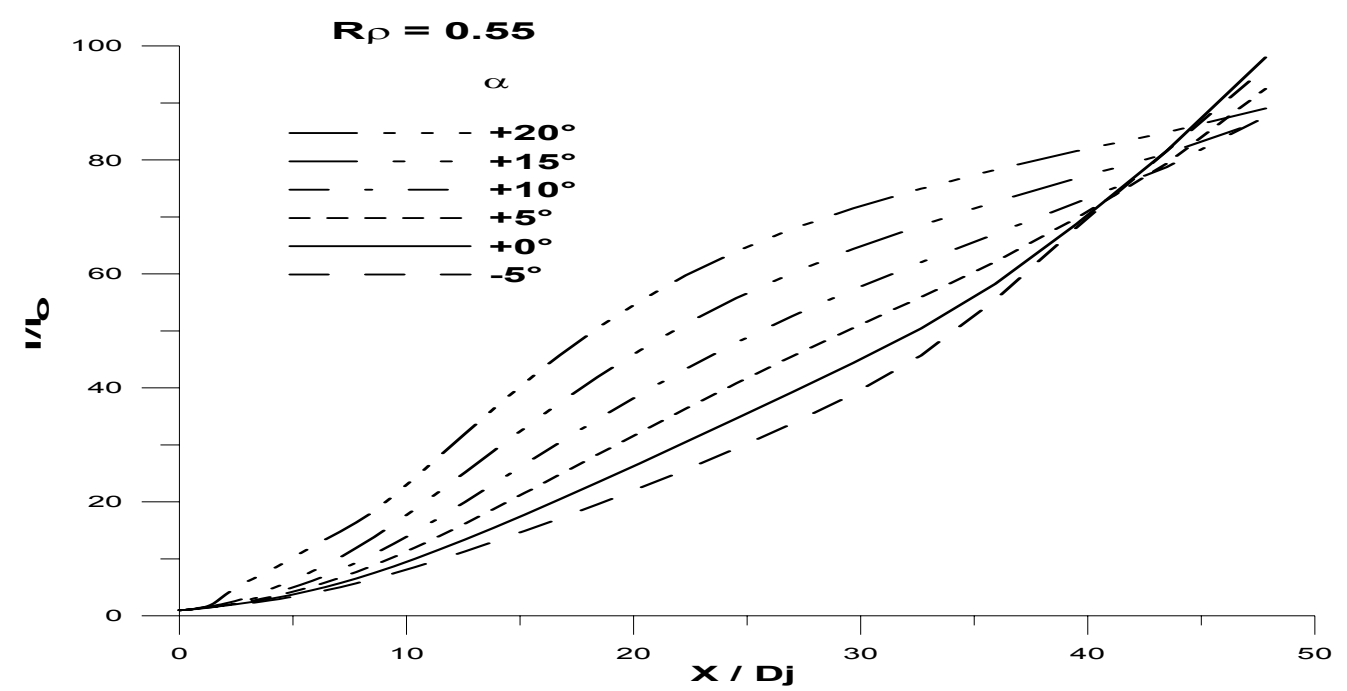

Fig. 6 Effects of deviation co-flow one the evolution of the mixing efficiency.

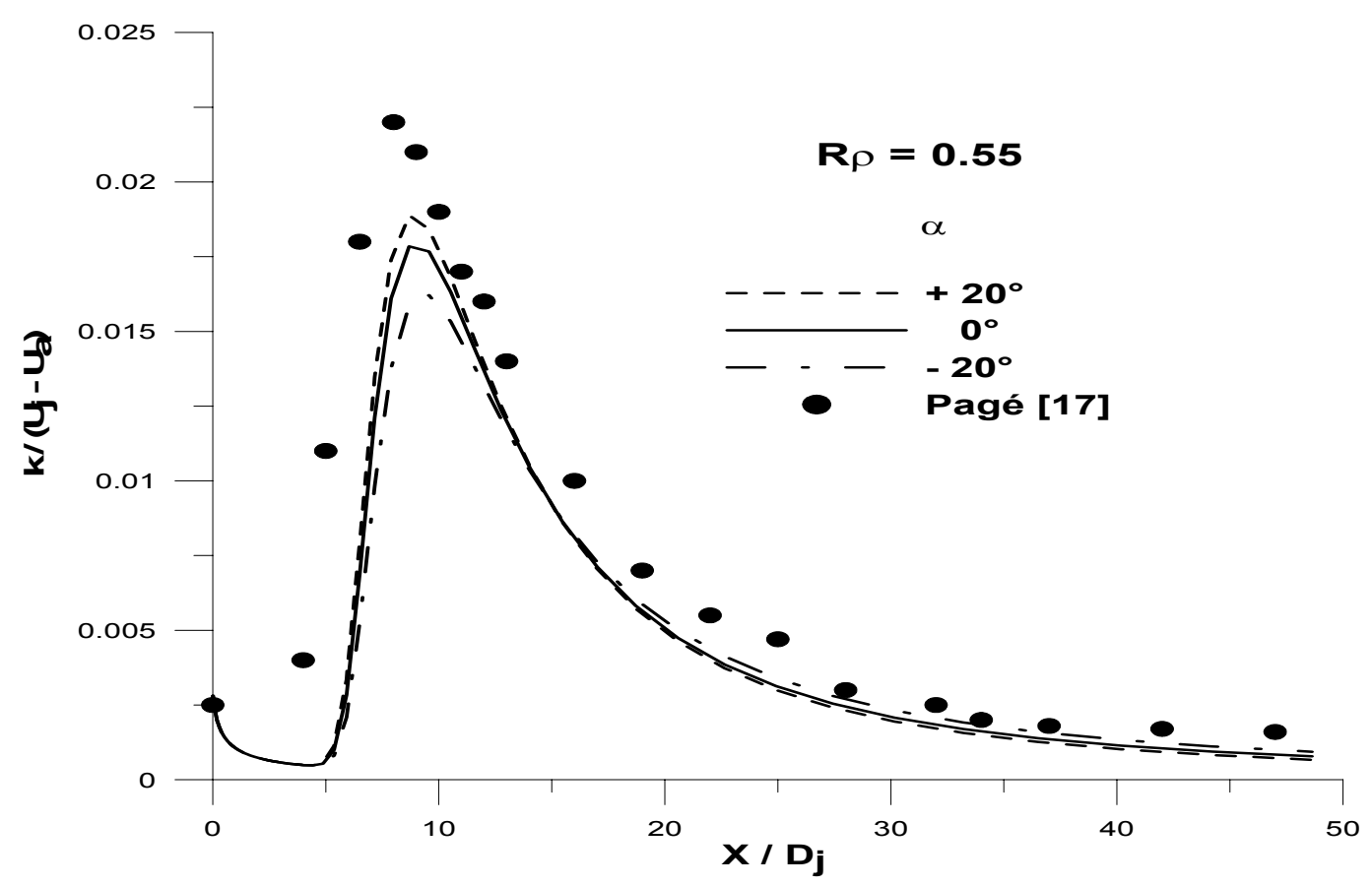

Fig.7 the centreline turbulent kinetic energy . 